Mathematical Modelling And Analysis

Volume 19 Number 2, April 2014, 169-179

http://dx.doi.org/10.3846/13926292.2014.909896

(c) Vilnius Gediminas Technical University, 2014
Publisher: Taylor\&Francis and VGTU

http://www.tandfonline.com/TMMA

Print ISSN: 1392-6292

Online ISSN: 1648-3510

\title{
Extinction and Non-Extinction of Solutions to a $p$-Laplace Equation with a Nonlocal Source and an Absorption Term
}

\section{Yuzhu Han, Haixia Li and Wenjie Gao}

Department of Mathematics, Jilin University

130012 Changchun, China

E-mail: yzhan@jlu.edu.cn

E-mail(corresp.): lihaixia0611@126.com

E-mail: wjgao@jlu.edu.cn

Received January 2, 2013; revised February 23, 2014; published online April 15, 2014

\begin{abstract}
In this short paper, the authors investigate the extinction and nonextinction of solutions to a fast diffusive $p$-Laplace equation with a nonlocal source and an absorption term. By applying the super-solution and sub-solution methods, instead of energy estimate methods, they give a classification of the exponents and coefficients for the solutions to vanish in finite time or not, which generalizes some previous results.
\end{abstract}

Keywords: $p$-Laplace equation, super-solution and sub-solution, extinction.

AMS Subject Classification: $35 \mathrm{~K} 59 ; 35 \mathrm{~K} 67$.

\section{Introduction}

In this paper, we consider a fast diffusive $p$-Laplace equation with a nonlocal source and an absorption term of the following form

$$
\begin{cases}u_{t}=\operatorname{div}\left(|\nabla u|^{p-2} \nabla u\right)+a \int_{\Omega} u^{q}(y, t) \mathrm{d} y-b u^{r}, & x \in \Omega, t>0, \\ u(x, t)=0, & x \in \partial \Omega, t>0, \\ u(x, 0)=u_{0}(x), & x \in \Omega,\end{cases}
$$

where $a, b, q, r>0,1<p<2, \Omega$ is a bounded domain in $R^{N}(N \geq 1)$ with smooth boundary $\partial \Omega$, and the initial datum $u_{0}(x)$ is a nonnegative nontrivial function such that $u_{0} \in L^{\infty}(\Omega) \cap W_{0}^{1, p}(\Omega)$.

The equation in (1.1) is a $p$-Laplace equation perturbed by both a nonlinear nonlocal source term and an absorption term, which describes the fast 
diffusion of the concentration of some non-Newtonian fluids through a porous medium or the temperature of some combustible substance (see $[3,20,24])$. In particular, problem (1.1) is a possible model for the diffusion system of some biological species with human-controlled distribution where $u(x, t)$ represents the density of the species at position $x$ and time $t, \operatorname{div}\left(|\nabla u|^{p-2} \nabla u\right)$ portrays the mutation (which we view as a spreading of the characteristic), $-b u^{r}$ is the growth capacity of the species at location $x$ and time $t$, whereas $a \int_{\Omega} u^{q}(y, t) \mathrm{d} y$ denotes the human-controlled distribution. Nonlocal term is a way to express that the evolution of the species in a point of space depends not only on nearby density but also on the total amount of species because of the effects of spatial inhomogeneity $[2,5]$.

Extinction in finite time is a phenomenon whereby the evolution of some nontrivial initial datum $u_{0}(x)$ produces a nontrivial solution $u(x, t)$ in a time interval $0<t<T$, but $u(x, t) \equiv 0$ for almost every $(x, t) \in \Omega \times(T, \infty)$. In this case, $T$ is called the extinction time. As one of the most important properties of solutions of evolutionary equations, extinction in finite time of solutions has been intensively studied by several authors (see $[6,7,8,9,10,13,14,17,18,21]$ and references therein). In particular, there are also some works concerning extinction of solutions of problem (1.1) for special cases. For instance, Liu [14] studied (1.1) when $p=2$ and showed that $q=r$ is the critical extinction exponent of solutions for (1.1). In a recent paper [4], Fang and Xu investigated the case $r=1$, proved that $q=p-1$ is the critical extinction exponent of solutions of problem (1.1) and gave the precise decay estimates, by using the methods of energy estimates. Their results show that the linear absorption term does not change the critical extinction exponents. However, when the absorption term is nonlinear, i.e. when $r \neq 1$, the problem is much more challenging and there is no result in this direction. For more works concerning the extinction phenomenon of solutions of fast diffusion equations, readers may refer to $[11,12,16,19,22,23,25]$.

Motivated by the works mentioned above, instead of using the methods of energy estimates, we investigate the extinction and non-extinction properties of solutions to problem (1.1) by constructing suitable super and sub-solutions and give an almost complete classification of the exponents and coefficients for the solutions to vanish in finite time or not.

Throughout this paper, we denote by $\varphi(x)$ the unique positive solution of the following elliptic problem

$$
-\operatorname{div}\left(|\nabla \varphi|^{p-2} \nabla \varphi\right)=1, \quad x \in \Omega ; \quad \varphi(x)=0, \quad x \in \partial \Omega
$$

and set

$$
M=\max _{x \in \bar{\Omega}} \varphi(x) \quad \text { and } \quad \mu=\int_{\Omega} \varphi^{p-1}(x) \mathrm{d} x .
$$

By the strong maximum principle we know that $M, \mu>0$. In Section 2, we will give some notations and preliminaries and state our main results. The proofs of the main results will be presented in Section 3. 


\section{Preliminaries and Main Results}

Because of the singularity of the $p$-Laplace operator, problem (1.1) does not admit classical solutions in general. Therefore, we consider the nonnegative solutions of (1.1) in some weak sense. To state the definition of the weak solutions, we first introduce some notations which will be used throughout this paper:

$$
\begin{aligned}
& Q=\Omega \times(0, \infty), \quad Q_{T}=\Omega \times(0, T), \\
& E=\left\{u \in L^{2 q}\left(Q_{T}\right) \cap L^{2 r}\left(Q_{T}\right) ; u \in C\left([0, T] ; L^{2}(\Omega)\right), \nabla u \in L^{p}\left(Q_{T}\right)\right\}, \\
& \widetilde{E}=\left\{u \in L^{2}\left(Q_{T}\right) ; \quad \frac{\partial u}{\partial t} \in L^{2}\left(Q_{T}\right), \nabla u \in L^{p}\left(Q_{T}\right) ;\left.u\right|_{\partial \Omega}=0\right\} .
\end{aligned}
$$

Definition 1. A nonnegative measurable function $u \in E$ is called a weak subsolution of problem (1.1) provided that for any $T>0$ and any $0 \leq \xi \in \widetilde{E}$ the following conditions hold:

$$
u(x, 0) \leq u_{0}(x), \quad x \in \Omega ; \quad u(x, t) \leq 0, \quad(x, t) \in \partial \Omega \times(0, T),
$$

and for $(x, t) \in Q_{T}$

$$
\begin{aligned}
& \int_{\Omega} u(x, T) \xi(x, T) \mathrm{d} x-\int_{\Omega} u_{0}(x) \xi(x, 0) \mathrm{d} x \\
& \quad \leq \iint_{Q_{T}}\left[u \frac{\partial \xi}{\partial t}-|\nabla u|^{p-2} \nabla u \nabla \xi+\left(a \int_{\Omega} u^{q}(y, t) \mathrm{d} y-b u^{r}\right) \xi(x, t)\right] \mathrm{d} x \mathrm{~d} t .
\end{aligned}
$$

The definition of a weak super-solution follows by replacing ' $\leq$ ' by ' $\geq$ ' in the above inequalities. Furthermore, if $u$ is a weak super-solution as well as a weak sub-solution, then we call it a weak solution of problem (1.1).

Local existence of weak solutions of (1.1) can be obtained by utilizing the methods of standard regularization (see [10]) or by applying the famous Schaefer's fixed point theorem (see [11]). Before proving our main results, we give a comparison principle for the super-solutions and sub-solutions of problem (1.1), which is similar to Proposition 2.3 in [10] and can be proved by a rather standard method as that in $[1,11,18,21]$.

Proposition 1. Let $u$ and $v$ be a nonnegative subsolution and a nonnegative supersolution of (1.1), respectively. If either $q \geq 1$ and $u$ is bounded from above or $0<q<1$ and $v$ has a positive lower bound, then $u(x, t) \leq v(x, t)$ in $Q_{T}$.

The main results of this paper read as follows.

Theorem 1. If $q>p-1$, then any solution $u(x, t)$ vanishes in finite time for suitably small initial data $u_{0}(x)$. If $q=p-1$ and a $<1$, then $u(x, t)$ vanishes in finite time for any nonnegative bounded initial data.

Theorem 2. If $r<\min \{q, 1\}$ or $r=q<1$ with $a|\Omega|<b$, then the solution $u(x, t)$ of problem (1.1) vanishes in finite time for appropriately small initial data $u_{0}(x)$. 
Theorems 1 and 2 show that when the source term is suitably weak or the absorption term is in some sense strong, solutions of problem (1.1) vanish in finite time.

Theorem 3. If $q<\min \{r, p-1\}$ or $r=q<p-1$ with $a \gamma>b$, then problem (1.1) admits at least one nonextinction solution for any nonnegative initial data. Here $\gamma>0$ will be given in the proof.

Theorem 4. If $q=p-1<r$ with a $>>1$, then problem (1.1) admits at least one non-extinction solution for any nonnegative initial data.

Theorems 3 and 4 tell us that when the source term is appropriately strong, problem (1.1) admits non-extinction solutions.

Theorem 5. If $q=p-1<r<1$ and $a|\Omega| \leq \lambda_{1}$, then $u(x, t)$ vanishes in the sense that $\lim _{t \rightarrow+\infty}\|u(\cdot, t)\|_{2}=0$. If $q=p-1<r<1$ with $a \mu=1$, then all the solutions $u(x, t)$ of problem (1.1) vanish in finite time for any nonnegative initial data. If $q=p-1<1 \leq r$ with $a \mu=1$, then (1.1) admits at least one non-extinction solution $u(x, t)$ for any positive initial data $u_{0}(x)$.

Theorem 5 shows that for the "critical" case $q=p-1$ with $a \mu=1$, whether the solution of (1.1) vanishes or not depends heavily on the absorption term.

Remark 1. It can be seen from Theorems $1,3,4$ and 5 that $q=p-1$ is the critical extinction exponent when the absorption is $\operatorname{linear}(r=1)$, which is consistent with the result obtained in [4] by using the integral estimate methods.

\section{Proof of the Main Results}

Proof of Theorem 1. The results of this theorem can be obtained by applying the comparison argument since the solutions of (1.1) is a subsolution of the problem in [11] with $m=1$. We only sketch the outlines for completeness.

Case (i): $q=p-1$ with $a \mu<1$. For any bounded smooth domain $\Omega^{\prime}$ such that $\Omega^{\prime} \supset \supset \Omega$, let $\phi(x)$ be the unique solution of the following elliptic problem

$$
\begin{cases}-\operatorname{div}\left(|\nabla \phi|^{p-2} \nabla \phi\right)=1, & x \in \Omega^{\prime}, \\ \phi(x)=0, & x \in \partial \Omega^{\prime} .\end{cases}
$$

By the comparison principle for the $p$-Laplace type elliptic problem we know $\varphi(x) \leq \phi(x)$ in $\Omega$. Set $\mu_{1}=\int_{\Omega} \phi^{p-1}(x) \mathrm{d} x, M_{1}=\max _{x \in \overline{\Omega^{\prime}}} \phi(x)$ and $\delta=$ $\min _{x \in \bar{\Omega}} \phi(x)$. It is known from the strong maximum principle that $\delta>0$.

We can choose a suitable domain $\Omega^{\prime} \supset \supset \Omega$ such that $a \mu_{1}<1$ by continuity. Define $v(x, t)=g(t) \phi(x)$, where $g(t)$ satisfies

$$
\left\{\begin{array}{l}
g^{\prime}(t) M_{1}+\left(1-a \mu_{1}\right) g^{p-1}(t)=0, \quad t>0 \\
g(0)=A \geq \delta^{-1}\left\|u_{0}\right\|_{L^{\infty}(\Omega)} .
\end{array}\right.
$$


Since $0<p-1<1$, it follows from the theories in ODEs that $g(t)$ is nonincreasing and $g(t)=0$ for all

$$
t \geq T^{*}=\frac{M_{1}}{\left(1-a \mu_{1}\right)(2-p)} A^{2-p} .
$$

Then it can be verified that for any $0<T<T^{*}, v(x, t)$ is a super-solution of (1.1) in $Q_{T}$. In fact, because $q=p-1$ and $g^{\prime}(t) \leq 0$, we know that $v(x, t)$ satisfies the following inequalities (in the weak sense)

$$
\begin{aligned}
\frac{\partial v}{\partial t} & -\operatorname{div}\left(|\nabla v|^{p-2} \nabla v\right)-a \int_{\Omega} v^{p-1}(y, t) \mathrm{d} y+b v^{r} \\
& =g^{\prime}(t) \phi(x)+g^{p-1}(t)-a \mu_{1} g^{p-1}(t)+b g^{r}(t) \phi^{r}(x) \\
& \geq g^{\prime}(t) M_{1}+\left(1-a \mu_{1}\right) g^{p-1}(t)=0 .
\end{aligned}
$$

In addition, $v(x, t) \geq 0$ on $\partial \Omega \times(0, T)$ for any $T>0$, and $v(x, 0) \geq u_{0}(x)$ by the choice of $A$. Moreover, there exists a positive constant $C_{1}$ such that $v(x, t) \geq C_{1}$ in $Q_{T}$. Therefore, by applying Proposition 1 to $(1.1)$ we see that $u(x, t) \leq$ $v(x, t)$ for $(x, t) \in Q_{T}$, which implies $u(x, T) \leq v(x, T)$. The arbitrariness of $T<T^{*}$ and $v\left(x, T^{*}\right)=0$ ensure that $u\left(x, T^{*}\right)=0$. Furthermore, let $\widetilde{u}(x, t)=u\left(x, t+T^{*}\right)$, then $\widetilde{u}(x, t)$ satisfies (1.1) with the initial condition $\widetilde{u}(x, 0)=0$. By the aforementioned proof, we see that $\widetilde{u}(x, t) \leq v(x, t)$ with any $A>0$. From the relation of the extinction time $T^{*}$ of $v(x, t)$ to $A$, it follows that $\widetilde{u}(x, t)=0$ for any $t>0$, i.e. $u(x, t)=0$ for any $t \geq T^{*}$.

Case (ii): $q>p-1$. Let $\phi(x)$ and $M_{1}$ be defined as in Case (i) and denote $k_{0}=\max \left\{1, \frac{2 M_{1}^{q-(p-1)} \mu}{\int_{\Omega} \phi^{q}(x) \mathrm{d} x}\right\}$. Set $v(x, t)=k \phi(x)$ with $k=\left(\frac{1}{a k_{0} \int_{\Omega} \phi^{q}(x) \mathrm{d} x}\right)^{\frac{1}{q-(p-1)}}$, then it is easy to verify that $v(x, t)$ is a supersolution of (1.1) when $u_{0}$ is small enough such that $u_{0}(x) \leq k \phi(x)$ in $\Omega$. Applying Proposition 1 to problem (1.1) in $Q_{T}$ for any $T>0$ we obtain that $u(x, t) \leq v(x, t)$ in $Q_{T}$, which implies that $u(x, t) \leq k M_{1}$. Therefore, $u(x, t)$ satisfies

$$
u_{t}-\operatorname{div}\left(|\nabla u|^{p-2} \nabla u\right)+b u^{r} \leq a\left(k M_{1}\right)^{q-(p-1)} \int_{\Omega} u^{p-1}(y, t) \mathrm{d} y, \quad x \in \Omega, t>0 .
$$

By the choice of $k$ and $k_{0}$ it is easily seen that $a\left(k M_{1}\right)^{q-(p-1)} \mu \leq \frac{1}{2}<1$. Therefore, by the results of Case (i), we can conclude that the solution $u(x, t)$ vanishes in finite time. The proof of this theorem is complete.

Proof of Theorem 2. We first prove the case $r=q$ with $b>a|\Omega|$. Set $v(x, t)=$ $g(t)$ where $g(t)$ satisfies the following ordinary differential equation

$$
\left\{\begin{array}{l}
g^{\prime}(t)+(b-a|\Omega|) g^{q}(t)=0, \quad t>0 \\
g(0)=\left\|u_{0}\right\|_{L^{\infty}(\Omega)}
\end{array}\right.
$$

Since $0<q<1$ and $b>a|\Omega|$, we know from the theories in ODEs that $g(t)$ vanishes at some finite time $T^{*}$. Moreover, as in the proof of Theorem 1 , it can be verified that $g(t)$ is a super-solution of (1.1). Thus, by applying 
Proposition 1 to $u(x, t)$ and $g(t)$ for any $0<T<T^{*}$ we know that $u(x, t)$ also vanishes at $T^{*}$.

In the case $r<\min \{q, 1\}$, let $g(t)$ satisfy the following ODE

$$
\left\{\begin{array}{l}
g^{\prime}(t)+\left(b-a|\Omega| g^{q-r}(t)\right) g^{r}(t)=0, \quad t>0, \\
g(0)=g_{0},
\end{array}\right.
$$

where $0<g_{0}<\left(\frac{b}{a|\Omega|}\right)^{\frac{1}{q-r}}$. Similar to the first case, it is known that $g(t)$ vanishes in finite time and $g(t)$ is a super-solution of problem (1.1) if $u_{0}(x)$ is so small that $\left\|u_{0}\right\|_{L^{\infty}(\Omega)} \leq g_{0}$. Applying Proposition 1 to $u(x, t)$ and $g(t)$ guarantees the finite time extinction of $u(x, t)$.

Remark 2. The hypotheses that $u_{0}$ is small in Theorems 1 and 2 can be removed if $a$ is suitably small.

Proof of Theorem 3. (i). Consider first the case $q<r \leq p-1$. Let $\lambda_{1}$ be the first eigenvalue of the following eigenvalue problem

$$
\begin{cases}-\operatorname{div}\left(|\nabla \psi|^{p-2} \nabla \psi\right)=\lambda|\psi|^{p-2} \psi, & x \in \Omega, \\ \psi(x)=0, & x \in \partial \Omega,\end{cases}
$$

and $\psi_{1}(x)>0(x \in \Omega)$ be the corresponding eigenfunction. We may normalize $\psi_{1}(x)$ such that $\left\|\psi_{1}\right\|_{L^{\infty}(\Omega)}=1$. Denote $\gamma=\int_{\Omega} \psi_{1}^{q}(x) \mathrm{d} x$ and let $g(t)$ satisfy the ODE problem

$$
\begin{cases}g^{\prime}(t)=-\lambda_{1} g^{p-1}(t)+a \gamma g^{q}(t)-b g^{r}(t), & t>0 \\ g(0)=0, & t>0 . \\ g(t)>0, & t>0 .\end{cases}
$$

It is easy to check that $g(t)$ is nondecreasing and bounded from above by $\min \left\{\left(\frac{a \gamma}{\lambda_{1}}\right)^{\frac{1}{p-1-q}},\left(\frac{a \gamma}{b}\right)^{\frac{1}{r-q}}\right\}$. Set $v(x, t)=\rho g(t) \psi_{1}(x)$. We shall show that $v(x, t)$ is a sub-solution of (1.1) when $\rho>0$ is sufficiently small. In fact, simple computations show that

$$
\frac{\partial v}{\partial t}=\rho\left(-\lambda_{1} g^{p-1}(t)+a \gamma g^{q}(t)-b g^{r}(t)\right) \psi_{1}(x)
$$

and

$$
\begin{aligned}
& \operatorname{div}\left(|\nabla v|^{p-2} \nabla v\right)+a \int_{\Omega} v^{q}(y, t) \mathrm{d} y-b v^{r} \\
& \quad=-\lambda_{1} \rho^{p-1} g^{p-1} \psi_{1}+a \gamma \rho^{q} g^{q}-b \rho^{r} g^{r} \psi_{1}^{r} .
\end{aligned}
$$

Denote $C=\max _{t>0}\left\{\lambda_{1} g^{p-1-q}(t), b g^{r-q}(t)\right\}$. Then for $v(x, t)$ to be a sub-solution of (1.1), it suffices to show that

$$
\lambda_{1} g^{p-1}(t) \rho^{p-1}+b g^{r}(t) \rho^{r} \leq a \gamma g^{q}(t)\left(\rho^{q}-\rho\right),
$$


which follows from

$$
C\left(\rho^{p-1}+\rho^{r}\right) \leq a \gamma\left(\rho^{q}-\rho\right)
$$

or equivalently,

$$
C\left(\rho^{p-1-q}+\rho^{r-q}\right) \leq a \gamma\left(1-\rho^{1-q}\right) .
$$

Since $q<r \leq p-1<1$, (3.6) holds for sufficiently small $\rho>0$.

Next, we turn our attention to construct a super-solution of (1.1). Set $w(x, t)=L$, where $L=\max \left\{\left\|u_{0}\right\|_{L^{\infty}(\Omega)},\left(\frac{a|\Omega|}{b}\right)^{\frac{1}{r-q}}, \rho \max _{t \geq 0} g(t)\right\}$. Then it is not hard to see that $w(x, t)$ is a super-solution and $v(x, t) \leq w(x, t)$. Therefore, by an iteration process, one can obtain a solution of problem (1.1), which satisfies $v(x, t) \leq u(x, t) \leq w(x, t)$. Because $v(x, t)$ does not vanish, neither does $u(x, t)$.

(ii). The case $q<p-1<r$ can be treated similarly to Case (i).

(iii). Finally we consider the case $r=q<p-1$ with $a \gamma>b$. Let $g(t)$ satisfy the following ODE

$$
\begin{cases}g^{\prime}(t)=-\lambda_{1} g^{p-1}(t)+(a \gamma-b) g^{q}(t), & t>0 \\ g(0)=0, & t>0 . \\ g(t)>0, & t>0 .\end{cases}
$$

Then $g(t)$ is nondecreasing and satisfies $g(t) \leq\left(\frac{a \gamma-b}{\lambda_{1}}\right)^{\frac{1}{p-1-q}}$. As in the proof of Case (i), we can construct a non-extinction sub-solution $v(x, t)=\rho g(t) \psi(x)$ with $\rho>0$ sufficiently small.

To construct a super-solution, consider the following eigenvalue problem

$$
\begin{cases}-\operatorname{div}\left(|\nabla \psi|^{p-2} \nabla \psi\right)=\lambda|\psi|^{p-2} \psi, & x \in \widetilde{\Omega} \\ \psi(x)=0, & x \in \partial \widetilde{\Omega}\end{cases}
$$

where $\widetilde{\Omega} \supset \Omega$ is a bounded domain with smooth boundary $\partial \widetilde{\Omega}$. Let $\widetilde{\lambda_{1}}$ and $\widetilde{\psi_{1}}(x)>0(x \in \widetilde{\Omega})$ be its first eigenvalue and the corresponding eigenfunction, respectively. We may normalize $\widetilde{\psi_{1}}(x)$ such that $\left\|\widetilde{\psi_{1}}\right\|_{L^{\infty}(\widetilde{\Omega})}=1$. Denote $\widetilde{\gamma}=\int_{\Omega}{\widetilde{\psi_{1}}}^{q}(x) \mathrm{d} x$ and $\widetilde{\delta}=\min _{x \in \bar{\Omega}} \widetilde{\psi_{1}}(x)>0$.

Set $w(x)=k \widetilde{\psi_{1}}(x)$. We shall show that $w(x)$ is a supersolution of problem (1.1) provided that $k>0$ is suitably large. Indeed, if $k=\max \left\{\left(\frac{a \widetilde{\gamma}}{\widetilde{\lambda_{1}} \widetilde{\delta}^{p-1}}\right)^{\frac{1}{p-1-q}}\right.$, $\left.\widetilde{\delta}^{-1}\left\|u_{0}\right\|_{L^{\infty}(\Omega)}, \rho \widetilde{\delta}^{-1}\left(\frac{a \gamma-b}{\lambda_{1}}\right)^{\frac{1}{p-1-q}}\right\}$, we know that $w(x) \geq 0$ on $\partial \Omega \times(0, \infty)$, $w(x) \geq u_{0}(x)$ in $\Omega$ and $w(x)$ satisfies the following inequalities (in the weak sense)

$$
\begin{aligned}
\frac{\partial w}{\partial t} & -\operatorname{div}\left(|\nabla w|^{p-2} \nabla w\right)-a \int_{\Omega} w^{q}(x, t) \mathrm{d} x+b w^{q} \\
& =k^{p-1} \widetilde{\lambda_{1}}{\widetilde{\psi_{1}}}^{p-1}(x)-a k^{q} \widetilde{\gamma}+b k^{q}{\widetilde{\psi_{1}}}^{q}(x) \\
& \geq k^{q}\left(k^{p-1-q} \widetilde{\lambda_{1}} \widetilde{\delta}^{p-1}-a \widetilde{\gamma}\right) \geq 0 .
\end{aligned}
$$

Moreover, $v(x, t) \leq w(x)$ by the choice of $k$. Therefore, by applying the monotonicity iteration we obtain a non-extinction solution $u(x, t)$ of $(1.1)$ satisfying $v(x, t) \leq u(x, t) \leq w(x)$. This completes the proof of Theorem 3 . 
Proof of Theorem 4. The proof of this theorem is similar to that of Theorem 3, so we only sketch the outline here. Set $v(x, t)=\rho g(t) \varphi(x)$ where $\varphi(x)$ is defined in (1.2) and $g(t)$ satisfies the following ODE problem

$$
\begin{cases}g^{\prime}(t)=M^{-1}\left\{(a \mu-1) g^{p-1}(t)-b M^{r} g^{r}(t)\right\}, & t>0, \\ g(0)=0, & t>0 . \\ g(t)>0, & \end{cases}
$$

Since $a \mu>1$ and $p-1<r$, it is known that $g(t)$ is nondecreasing and is bounded from above by $\left(\frac{a \mu-1}{b M^{r}}\right)^{\frac{1}{r-(p-1)}}$. Then $v(x, t)$ is a sub-solution of (1.1) if $\rho>0$ is sufficiently small. On the other hand, the super-solution $w(x, t)$ can be chosen to be a large positive constant $L$ satisfying $L \geq \max \left\{\left\|u_{0}\right\|_{L^{\infty}(\Omega)},\left(\frac{a|\Omega|}{b}\right)^{\frac{1}{r-q}}\right.$, $\left.\rho M \max _{t \geq 0} g(t)\right\}$. It can be observed that $(v, w)$ is a pair of sub-solution and super-solution of (1.1) satisfying $v(x, t) \leq w(x, t)$. Therefore, by monotonicity iteration, we know that (1.1) admits at least one solution $u(x, t)$ such that $v(x, t) \leq u(x, t) \leq w(x, t)$. Since $v(x, t)>0$ in $\Omega \times(0,+\infty), u(x, t)$ cannot vanish at any finite time. The proof of Theorem 4 is complete.

Proof of Theorem 5. (i). Let $u(x, t)$ be any solution of (1.1). It can be verified that, for the case $p-1=q<r<1$, a suitably large constant $L$ is a supersolution of (1.1). Therefore, we know that $u(x, t) \leq L$ in $\Omega \times(0,+\infty)$. Multiplying Equation (1.1) by $u$ and integrating by parts over $\Omega$ yield the identity

$$
\frac{1}{2} \frac{d}{d t} \int_{\Omega} u^{2} \mathrm{~d} x+\int_{\Omega}|\nabla u|^{p} \mathrm{~d} x+b \int_{\Omega} u^{r+1} \mathrm{~d} x=a \int_{\Omega} u^{p-1} \mathrm{~d} x \int_{\Omega} u \mathrm{~d} x .
$$

Recall the embedding theorem

$$
\int_{\Omega}|\nabla u|^{p} \mathrm{~d} x \geq \lambda_{1} \int_{\Omega} u^{p} \mathrm{~d} x .
$$

Combining this result with (3.9) and using Hölder's inequality on the right hand side of (3.9) one obtains

$$
\frac{1}{2} \frac{d}{d t} \int_{\Omega} u^{2} \mathrm{~d} x+\lambda_{1} \int_{\Omega} u^{p} \mathrm{~d} x+b \int_{\Omega} u^{r+1} \mathrm{~d} x \leq a|\Omega| \int_{\Omega} u^{p} \mathrm{~d} x .
$$

Noticing that $a|\Omega| \leq \lambda_{1}$ and $u(x, t) \leq L$, we see from (3.10) that

$$
\frac{d}{d t} \int_{\Omega} u^{2} \mathrm{~d} x+2 b L^{r-1} \int_{\Omega} u^{2} \mathrm{~d} x \leq 0,
$$

which implies

$$
\int_{\Omega} u^{2} \mathrm{~d} x \leq e^{-2 b L^{r-1} t} \int_{\Omega} u_{0}^{2} \mathrm{~d} x .
$$

This shows that $\|u(\cdot, t)\|_{2}$ tends to 0 exponentially as $t \rightarrow+\infty$. 
(ii). Next, let us prove the extinction results of the case $q=p-1<r<1$. Set $w(x, t)=g(t) \varphi(x)$, where $g(t)$ satisfies

$$
\left\{\begin{array}{l}
g^{\prime}(t)+b M^{r-1} g^{r}(t)=0, \quad t>0, \\
g(0)=A>0 .
\end{array}\right.
$$

Since $0<r<1, g(t)$ is nonincreasing and vanishes at the finite time $T^{*}=$ $\frac{A^{1-r}}{b M^{r-1}(1-r)}$ for any $A>0$. Noticing $q=p-1$ and $a \mu=1$, one can check that $w(x, t)$ is a super-solution of (1.1) provided that $A>0$ is appropriately large such that $A \varphi(x) \geq u_{0}(x)$ in $\Omega$. Now by using the arguments similar to that of the proof of Theorem 1, we know that every solution $u(x, t)$ of $(1.1)$ vanishes at a time no later than $T^{*}$.

(iii). Finally we consider the case $q=p-1<1 \leq r$. First we construct a non-extinction sub-solution of (1.1). Set $v(x, t)=h_{0} e^{-\alpha t} \varphi(x)$, where $h_{0}, \alpha$ are two positive constants to be determined. Noticing that $a \mu=1$, it is easily checked that when $r=1, v(x, t)$ is a sub-solution of (1.1) provided that $\alpha \geq b$ and $h_{0}$ is so small such that $h_{0} \varphi(x) \leq u_{0}(x)$. When $r>1$, for $v(x, t)$ to be sub-solution of (1.1) it is reasonable to choose first $h_{0}$ so small such that $h_{0} \varphi(x) \leq u_{0}(x)$ and then $\alpha \geq b h_{0}^{r-1} M^{(r-1)}$. Next, since $r>q$ and $v(x, t)$ is bounded, we can choose a sufficiently large constant $L \geq v(x, t)$ to be a supersolution of (1.1). Therefore, by monotonicity iteration, one obtains a solution $u(x, t)$ of $(1.1)$ satisfying $v(x, t) \leq u(x, t) \leq L$. Since $v(x, t)$ does not vanish at any finite time, neither does $u(x, t)$. The proof of Theorem 5 is complete.

Remark 3. According to Theorems 1, 4 and the first part of Theorem 5 it is easy to see that $q=p-1$ is the critical extinction exponent for problem (1.1) when $r=1$, which is consistent with the results obtained in [4]. However, when $r=1$ and $q=p-1$, for the nonlocal problem under consideration, the first eigenvalue $\lambda_{1}$ of $p$-Laplacian in $\Omega$ no longer plays the same role as it does in the local case (see Theorem 1 in [15]).

Remark 4. There still exist some gaps of the coefficients in which case we do not know whether problem (1.1) admits non-extinction solutions or not, due to technique reasons. For example, combining Theorem 2 with Theorem 3 one observes that there is no result on whether there exists a non-extinction solution of problem (1.1) or not when $q=r<p-1$ with $\frac{b}{|\Omega|} \leq a \leq \frac{b}{\gamma}$ (noticing by the definition of $\gamma$ that $\gamma<|\Omega|)$.

\section{Acknowledgement}

This project is supported by NSFC (11271154), by Key Lab of Symbolic Computation and Knowledge Engineering of Ministry of Education and by the 985 program of Jilin University. The authors highly appreciate the referees for their valuable comments which improve the original manuscript. 


\section{References}

[1] J.R. Anderson. Local existence and uniqueness of solutions of degenerate parabolic equations. Comm. Partial Differential Equations, 16(1):105-143, 1991. http://dx.doi.org/10.1080/03605309108820753.

[2] A. Calsina, C. Perello and J. Saldana. Non-local reaction-diffusion equations modelling predator-prey coevolution. Publ. Mat., 38(2):315-325, 1994. http://dx.doi.org/10.5565/PUBLMAT_38294_04.

[3] E. DiBenedetto. Degenerate Parabolic Equations. Springer, 1993.

[4] Z.B. Fang and X.H. Xu. Extinction behavior of solutions for the p-Laplacian equations with nonlocal sources. Nonlinear Anal. Real World Appl., 13(4):17801789, 2012. http://dx.doi.org/10.1016/j.nonrwa.2011.12.008.

[5] J. Furter and M. Grinfeld. Local vs. non-local interactions in population dynamics. J. Math. Biol., 27(1):65-80, 1989. http://dx.doi.org/10.1007/BF00276081.

[6] V.A. Galaktionov, L.A. Peletier and J.L. Vazquez. Asymptotics of the fastdiffusion equation with critical exponent. SIAM J. Math. Anal., 31(5):11571174, 2000. http://dx.doi.org/10.1137/S0036141097328452.

[7] V.A. Galaktionov and J.L. Vazquez. Asymptotic behaviour of nonlinear parabolic equations with critical exponents. A dynamical systems approach. $J$. Funct. Anal., 100(2):435-462, 1991. http://dx.doi.org/10.1016/0022-1236(91)90120-T.

[8] V.A. Galaktionov and J.L. Vazquez. Extinction for a quasilinear heat equation with absorprtion I. Technique of intersection comparison. Comm. Partial Differential Equations, 19(7-8):1075-1106, 1994. http://dx.doi.org/10.1080/03605309408821046.

[9] V.A. Galaktionov and J.L. Vazquez. Extinction for a quasilinear heat equation with absorption II. A dynamical systems approach. Comm. Partial Differential Equations, 19(7-8):1107-1137, 1994. http://dx.doi.org/10.1080/03605309408821047.

[10] Y.Z. Han and W.J. Gao. Extinction for a fast diffusion equation with a nonlinear nonlocal source. Arch. Math., 97(4):353-363, 2011. http://dx.doi.org/10.1007/s00013-011-0299-1.

[11] Y.Z. Han and W.J. Gao. Extinction and non-extinction for a polytropic filtration equation with a nonlocal source. Appl. Anal., 92(3):636-650, 2013. http://dx.doi.org/10.1080/00036811.2011.632766.

[12] C.H. Jin, J.X. Yin and Y.Y. Ke. Critical extinction and blow-up exponents for fast diffusive polytropic filtration equation with sources. Proc. Edinb. Math. Soc., 52:419-444, 2009. http://dx.doi.org/10.1017/S0013091507000399.

[13] G. Leoni. A very singular solution for the porous media equation $u_{t}=$ $\Delta u^{m}-u^{p}$ when $0<m<1$. J. Differential Equations, 132(2):353-376, 1996. http://dx.doi.org/10.1006/jdeq.1996.0184.

[14] W.J. Liu. Extinction and non-extinction of solutions for a nonlocal reactiondiffusion problem. Electron. J. Qual. Theory Differ. Equ., 15:1-12, 2010.

[15] W.J. Liu. Extinction properties of solutions for a class of fast diffusive pLaplacian equations. Nonlinear Anal., 74(13):4520-4532, 2011.

http://dx.doi.org/10.1016/j.na.2011.04.016. 
[16] W.J. Liu, M.X. Wang and B. Wu. Extinction and decay estimates of solutions for a class of porous medium equations. J. Inequal. Appl., 2007, 2007.

[17] W.J. Liu and B. Wu. A note on extinction for fast diffusive p-Laplacian with sources. Math. Methods Appl. Sci., 31(12):1383-1386, 2008. http://dx.doi.org/10.1002/mma.976.

[18] Y. Tian and C.L. Mu. Extinction and non-extinction for a p-Laplacian equation with nonlinear source. Nonlinear Anal., 69(8):2422-2431, 2008. http://dx.doi.org/10.1016/j.na.2007.08.021.

[19] Y.F. Wang and J.X. Yin. Critical extinction exponents for a polytropic filtration equation with absorption and source. Math. Methods Appl. Sci., 2012.

[20] Z.Q. Wu, J.N. Zhao, J.X. Yin and H.L. Li. Nonlinear Diffusion Equations. World Scientific Publishing Company Incorporated, 2001.

[21] J.X. Yin and C.H. Jin. Critical extinction and blow-up exponents for fast diffusive p-Laplacian with sources. Math. Methods Appl. Sci., 30(10):1147-1167, 2007. http://dx.doi.org/10.1002/mma.833.

[22] J.X. Yin, J. Li and C.H. Jin. Non-extinction and critical exponent for a polytropic filtration equation. Nonlinear Anal., 71(1):347-357, 2009. http://dx.doi.org/10.1016/j.na.2008.10.082.

[23] H.J. Yuan, S.Z. Lian, W.J. Gao, X.J. Xu and C.L. Cao. Extinction and positivity for the evolution p-Laplacian equation in Rn. Nonlinear Anal., 60(6):1085-1091, 2005. http://dx.doi.org/10.1016/j.na.2004.10.009.

[24] J.N. Zhao. Existence and nonexistence of solutions for existence and nonexistence of solution for $u_{t}=\operatorname{div}\left(|\nabla u|^{p-2} \nabla u\right)+f(x, t, u, \nabla u)$. J. Math. Anal. Appl., 172(1):130-146, 1993. http://dx.doi.org/10.1006/jmaa.1993.1012.

[25] P. Zheng and C.L. Mu. Extinction and decay estimates of solutions for a polytropic filtration equation with the nonlocal source and interior absorption. Math. Methods Appl. Sci., 2012. 